\title{
The Laparoscopic Approach in the Treatment of Distal Colorectal Cancer
}

\author{
Alexander Lebedyev ${ }^{1}$, Damien Urban ${ }^{2}$, Danny Rosin ${ }^{1}$, Amram Ayalon ${ }^{1}$, Dan Aderka ${ }^{2}$, Mordehai \\ Gutman $^{1}$ and Oded Zmora ${ }^{1, *}$
}

\author{
${ }^{1}$ Department of Surgery and Transplantation and ${ }^{2}$ Oncology Institute, Sheba Medical Center, Tel Hashomer, Affiliated \\ to the Faculty of Medicine, Tel Aviv University, Tel Aviv, Israel
}

\begin{abstract}
Purpose: The use of laparoscopic techniques has been widely accepted for most solid organ malignancies, but its use for distal colorectal cancer is still controversial. The aim of this study is to review our experience with laparoscopic resections of distal colorectal cancer and to assess patients' outcome.
\end{abstract}

\begin{abstract}
Methods: A retrospective chart review of prospectively entered data base was made to identify patients who underwent laparoscopic resections of distal colorectal cancer. Data relative to demographics, tumor characteristics, surgical procedure and adjuvant or neoadjuvant treatment were recorded. Long term follow up (survival and recurrence) was established from the patients' charts and telephone interviews.
\end{abstract}

Results: 94 consecutive patients underwent laparoscopic resections due to distal colorectal cancer. Surgery was in curative intent in 89 cases $(95 \%)$. Conversion rate was $20 \%$. Four patients $(4 \%)$ died from different septic complications in the early postoperative period. Complete long term follow up follow up was achieved in $71(75 \%)$ patients. There were no port site metastases. Local recurrence was diagnosed in 3 cases $(4 \%)$. Twenty one patients (30\%) died during this period, 11 due to metastatic disease, 1 from pneumonia, 9 patients from other non cancer related reasons.

Conclusions: Laparoscopic surgery may be safe and feasible for the treatment of distal colorectal cancer with acceptable long term oncologic results. The complexity of the procedure as well as the level of anastomosis results in relatively high complication rate, which may potentially improve by overcoming the "institutional learning curve". Randomized controlled trials comparing open and laparoscopic surgery for rectal cancer should verify these results, before the laparoscopic approach can be widely recommended.

Keywords: Anterior resection, Cancer, Laparoscopy, Rectum, Retrospective studies.

\section{INTRODUCTION}

Laparoscopic surgery has become part of the surgeon's toolbox for most abdominal organs, including surgery of the large bowel. Large trials have shown that laparoscopic right and left colon resections for the treatment of cancer result in adequate oncologic resection and equal cancer related survival when compared to open surgery. Laparoscopic colectomy have been shown to be associated with several short term benefits, such as improved cosmetic result, decreased postoperative pain, earlier recovery and possibly lower early complication rate. Laparoscopy may also be associated with several potential long term benefits such as fewer adhesions and decreased incisional hernias rate.

The surgical treatment of distal colorectal cancer is challenging irrespective of the surgical approach, and requires advanced surgical skills. This challenge is further emphasized with the laparoscopic technique, owing to the limited space at the pelvis, proximity to adjacent pelvic structures, the need to work in different quadrants of the

\footnotetext{
*Address correspondence to this author at the Department of Surgery and Transplantation, Sheba Medical Center, Tel-Hashomer 52621, Israel; Tel: (972-3) 530 2247; Fax: (972-3) 534 5826;

E-mail: ozmora@post.tau.ac.il
}

abdominal cavity, and difficulty in distal rectal transection with the currently available laparoscopic staplers. Owing to these difficulties, the adequacy of the laparoscopic approach for distal colorectal cancer is still controversial.

The aim of current study was review our experience with the laparoscopic approach for distal colorectal cancer, and assess the feasibility, safety and oncologic outcome of this procedure.

\section{METHODS}

A retrospective chart review of prospectively entered data-base of patients who underwent laparoscopic colon and rectal surgery was performed to identify those who had resection for distal colorectal cancer, defined as any tumor below the rectosigmoid junction. Data relative to demographic and clinical characteristics, surgical procedure, postoperative course, oncologic treatment and follow-up were collected from the medical charts. Long term follow up was established by telephone interview when required, and survival data were also cross-checked against the national census.

Preoperative evaluation routinely included computerized tomography (CT) of the abdomen and chest, rectoscopy and 
full colonoscopy, transrectal ultrasound for T staging for mid and low rectal cancer, and blood CEA levels. Preoperative chemoradiation $(50.4 \mathrm{G})$ was routinely given to patients with T3 mid and low rectal cancer, and selectively for lower third or high risk $\mathrm{T} 2$ tumors.

Surgical technique routinely included medial to lateral dissection, total mesorectal excision for mid and low rectal cancers, and transection $5 \mathrm{~cm}$ distal to the tumor in high rectal tumors, with transanal intracorporeal double stapled anastomosis. Diverting stoma was selectively used for coloanal anastomosis at the surgeon's decision.

All patients were examined at the outpatient clinic two weeks after discharge from the hospital, every three months during the first three years and every 6 months up to 5 years from surgery. Colonoscopy was performed at one year from surgery and repeated every three years postoperatively or on demand.

The study has been approved by the institutional ethics committee (IRB), and all patients who were interviewed by telephone gave their verbal consent prior to the interview.

\section{RESULTS}

Ninety four patients underwent laparoscopic resection due to distal colorectal cancer between 1997 and 2009 in our department. There were 51 males and 43 females, at a mean age of 68 years, all of which suffered from invasive adenocarcinoma of the rectum, from $1 \mathrm{~cm}$ up to $20 \mathrm{~cm}$ from the anal verge. In 89 (95\%) of the cases, the surgical procedure was defined as resection in curative intent. Neoadjuvant chemoradiation was used in 24 patients, and 33 received postoperative adjuvant therapy. In all cases, circumferential margins were negative for tumor. Demographic and clinical characteristic of the study group are listed in Table $\mathbf{1}$

In 81 cases reconstructive surgery was performed and 13 had abdominoperineal resection. Protective ileostomy was performed in $22(23 \%)$ cases. Mean operative time was 279 min, and mean postoperative hospital stay was 10 days. Positive Conversion to open surgery was required in $19(20 \%)$ patients. Reasons of conversion are listed in Table $\mathbf{2}$.

Early postoperative complications (up to 30 days from surgery) were diagnosed in 29 (31\%) patients, of which 13 $(13.5 \%)$ were considered major. The most common complication was anastomotic leak- $9(9.5 \%)$ patients. In 5 cases anastomotic leak occurred after anterior resection with colorectal anastomosis, and 4 were after total mesorectal excision with coloanal anastomosis. Anastomotic leak was an indication for reoperation in 6 patients, and in one case reoperation was performed due to missed small bowel injury. Clinical anastomotic leak was evident in 3 patients with protective ileostomy. Postoperative complications are elaborated in Table 3.

Perioperative mortality (within 30 days after surgery) was $4 \%$ (4 patients). In all cases death was attributed to septic complications due to anastomotic leak, two of which after coloanal anastomosis. All 4 mortalities occurred in elderly (72 years and older) patients with significant co-morbidities. In 3 of these patients preoperative oral mechanical bowel preparation was not used, as its routine use has been abundant in our department in the past decade.
Table 1. Demographic and Clinical Characteristics

\begin{tabular}{|c|c|}
\hline Overall Patients & 94 \\
\hline Male & $51(54 \%)$ \\
\hline Female & $43(46 \%)$ \\
\hline Mean age (years, range) & $68(37-92)$ \\
\hline \multicolumn{2}{|l|}{ ASA score: } \\
\hline 1 & $6(6 \%)$ \\
\hline 2 & $42(45 \%)$ \\
\hline 3 & $44(47 \%)$ \\
\hline 4 & $2(2 \%)$ \\
\hline \multicolumn{2}{|c|}{ Distance of the tumor from the anal verge: } \\
\hline $1-5 \mathrm{~cm}$ & $12(13 \%)$ \\
\hline $5-10 \mathrm{~cm}$ & $26(27 \%)$ \\
\hline $10-15 \mathrm{~cm}$ & $29(31 \%)$ \\
\hline $15-20 \mathrm{~cm}$ & $27(29 \%)$ \\
\hline \multicolumn{2}{|l|}{ Tumor Size: } \\
\hline$<3 \mathrm{~cm}$ & $43(46 \%)$ \\
\hline $3-5 \mathrm{~cm}$ & $29(31 \%)$ \\
\hline$>5 \mathrm{~cm}$ & $22(23 \%)$ \\
\hline \multicolumn{2}{|l|}{ Tumor Stage: } \\
\hline $\mathrm{T} 1$ & $16(17 \%)$ \\
\hline $\mathrm{T} 2$ & $29(31 \%)$ \\
\hline $\mathrm{T} 3$ & $45(48 \%)$ \\
\hline $\mathrm{T} 4$ & $4(4 \%)$ \\
\hline \multicolumn{2}{|c|}{ Number of harvested lymph nodes (mean (range)): } \\
\hline High anterior resection & $14(2-23)$ \\
\hline Low anterior resection & $12(2-40)$ \\
\hline Abdominoperineal resection & $8(2-30)$ \\
\hline \multicolumn{2}{|l|}{ N status: } \\
\hline N0 & $52(55 \%)$ \\
\hline N1 & $27(29 \%)$ \\
\hline N2 & $15(16 \%)$ \\
\hline Positive circumferential margins & $0(0 \%)$ \\
\hline \multicolumn{2}{|c|}{ Preoperative oral mechanical bowel preparation: } \\
\hline Yes & $32(34 \%)$ \\
\hline No & $62(66 \%)$ \\
\hline Neoadjuvant chemoradiation & $24(26 \%)$ \\
\hline Adjuvant chemotherapy & $33(35 \%)$ \\
\hline
\end{tabular}

As for long term follow up, we were able to obtain adequate full information on $71(75 \%)$ patients. Mean length of follow up was 42 months (range: 2-108). There were no port-site metastases in our series. Local recurrence was diagnosed in $3(4 \%)$ patients, 2 of which had adenocarcinoma of the upper rectum and 1 of the middle rectum.

Twenty one $(30 \%)$ patients died during the follow up period. Eleven died from metastatic disease, 1 from pneumonia, and 9 patients from other non cancer related reasons. Thus, at a mean follow up of 3.5 years, the overall survival of patients who underwent laparoscopic resection of distal 
colorectal cancer was $70 \%$, and the cancer related survival was $85 \%$. Four patients are alive with recurrent distant disease, resulting in overall disease free survival of $65 \%$.

Table 2. Reasons for Conversion

\begin{tabular}{|c|c|}
\hline & No. of patients (\%) \\
\hline \hline Overall conversion & $19(20 \%)$ \\
\hline Difficult dissection & $5(5 \%)$ \\
\hline Leak from the stapler line & $4(4 \%)$ \\
\hline Enterotomy & $2(2 \%)$ \\
\hline Adhesions to bladder & $2(2 \%)$ \\
\hline Ureternal injury & $1(1 \%)$ \\
\hline Intraabdominal bleeding & $3(3 \%)$ \\
\hline Need for intraoperative colonoscopy & $2(2 \%)$ \\
\hline
\end{tabular}

Table 3. Early Postoperative Complications

\begin{tabular}{|c|c|}
\hline & No. of patients (\%) \\
\hline \hline Overall complication & $29(31 \%)$ \\
\hline Anastomotic leak & $9(9.5 \%)$ \\
\hline Intrabdominal abscess & $3(3 \%)$ \\
\hline Missed small bowel injury & $1(1 \%)$ \\
\hline Paralytic ileus & $2(2 \%)$ \\
\hline Urinary retention & $4(4 \%)$ \\
\hline Perineal abscess (after APR) & $2(2 \%)$ \\
\hline Wound infection & $8(9 \%)$ \\
\hline
\end{tabular}

Long term complications included incisional hernia located at the extraction site in 11(15.5\%) patients, and small bowel obstruction which occurred in $5(7 \%)$ cases.

\section{DISCUSSION}

Most large multicenter randomized controlled trials comparing open and laparoscopic colectomy for the treatment of cancer excluded patients with the rectal tumors, owing to the complexity of laparoscopic surgery of the rectum. The British CLASICC trial [1] is the only exception, which included both patients with colon and rectal cancers. In this study, 293 patients (47\% of the entire study group) had surgery for rectal cancer, of which 160 underwent laparoscopic resection. There was a trend towards higher rate of involved circumferential resection margins in the laparoscopic group, and patients who required conversion had higher postoperative complication rate and longer hospital stay. The borderline difference in positive circumferential resection margin however, did not translate to a difference in three year local recurrence, disease-free, and overall survival rates.

Laparoscopic surgery for distal colorectal cancer requires both adequate colorectal training and mastering laparoscopic colorectal techniques. Specific obstacles associated with laparoscopic approach may include the need to work in different quadrants of the abdominal cavity, as adequate mobilization of the splenic flexure is frequently required, and difficulty in retraction of the rectum and other pelvic organs for adequate exposure. This may be more prominent in male patients with narrow pelvis, and with those with large tumors. Additionally, transection of the rectum at a right angle, especially at the anorectal junction level, may be difficult using the currently available laparoscopic staplers. On the other hand, the magnified view provided by the laparoscopic procedure, may allow more precise dissection, which was theoretically presumed to allow higher rate of nerve preservation $[2,3]$.

The results of this study suggest that the laparoscopic approach for the treatment distal colorectal cancer is feasible, and results in adequate oncologic resection, as measured by the parameters of number of harvested lymph nodes and distal and circumferential resection margins. Overall and disease free survival in our series favorably compare with previous series of open and laparoscopic resection of rectal cancer, adding validity to the oncologic safety of this procedure [4-6]. Conversion to open surgery was required in $20 \%$ of the patients in this series, which is significant, but in line with previous publications, and emphasizes that technical complexity of this procedure. We believe that this relatively high conversion rate also reflects the institutional learning curve with this complex procedure, when done by multiple surgeons, and conversion rate may decrease with more experience gained. Early complication rate, including anastomotic leak, was significant, although in line with previous publications. Any attempt to optimize operative and perioperative techniques should be made, to lower this significant complication rate $[2,3,7]$. Once again, this relatively high complication rate may be partially attributed to the institutional learning curve, and would hopefully improve with experience.

Perioperative techniques have significant impact on postoperative outcome, including early postoperative complication rate. Several perioperative methods however are currently controversial, including the routine use of preoperative mechanical bowel preparation and the routine use of diverting stoma for low colorectal and coloanal anastomosis. Several large scale multicenter studies as well as meta-analysis have shown that mechanical bowel preparation does not reduce anastomotic leak and other infectious complications after colon and rectal surgery. Most of these studies however, did not include significant number of patients with low colorectal or coloanal anastomosis. Bretagnol et al., [8] reported a case series of 52 consecutive cases of rectal surgery without preoperative mechanical bowel preparation compared to a matched group who did have preparation, and found lower complication rate without preparation. Van't Sant et al., [9], in a subgroup analyses of the Dutch multicenter trial on bowel preparation, analyzed 449 patients who had a resection with anastomosis below the peritoneal reflection, which were randomly assigned to mechanical bowel preparation or no preparation. The incidence of anastomotic leak was $7.6 \%$ for patients who received mechanical bowel preparation and $6.6 \%$ for patients who did not.

In the past decade, our department has adopted a policy of selective mechanical bowel preparation, avoiding its routine use, and for this reason, large portion of the patients in this study had laparoscopic resection of distal colorectal segments without mechanical bowel preparation [10]. Not surprisingly, large portion of the patients who had anasto- 
motic leak did not have mechanical bowel preparation, reflecting this policy. Despite strong evidence supporting omission of mechanical bowel preparation in colon surgery, the data for rectal surgery are still limited, especially concerning coloanal anastomosis. Specifically, if proximal diversion is planned, mechanical bowel preparation may be considered, to avoid column of stool which may lodge in the colon for extended periods. Randomized control trials assessing specifically the use of mechanical bowel preparation in patients undergoing coloanal anastomosis are required before firm conclusions can be made.

A second controversy is the need for routine use of proximal diversion in a low colorectal and coloanal anastomosis. Although diverting stoma may alleviate the clinical sequel of anastomotic leak, it does not prevent leak, and is associated with potential stoma or closure associated complications. In randomized, controlled pilot study Ulrich et al., [11] evaluated the need for diverting ileostomy in patients undergoing low anterior resection. The symptomatic anastomotic leakage rate was significantly higher in the nonstoma group (37.5\% Vs. 5.5\% $\mathrm{P}=0.02)$. Our policy is to divert in cases of hand-sewn coloanal anastomosis, difficult pelvic dissection, on patients following neoadjuvant therapy and in high risk patients due to significant past medical history [12,13]. Overall, $22(23 \%)$ patients in this series were diverted.

The best method for preoperative staging of patients with rectal cancer, as well as the indications for preoperative radiation therapy, are also an issue of controversy. Transrectal ultrasound is user dependent but cheaper and usually easily accessible, and is useful to differentiate between T2 and T3 lesions. Pelvic MRI is very precise in detecting circumferential margins at risk, and differentiates between $\mathrm{T} 3$ and $\mathrm{T} 4$ editions. MRI test is costly and may be less accessible. Since our practice is to offer preoperative radiation therapy to patients with T3 lesion or higher, we usually use transrectal ultrasound for staging. In our practice, MRI was additionally used in selected cases only. However, MRI is an excellent staging tool, and should be used especially in centers where circumferential margins at risk are the main indication for radiation.

In the past decade, the policy of our department has shifted towards the routine use of the laparoscopic approach for colon and rectal surgery unless contraindicated. The cases presented in this series were performed by a number of surgeons, some of which are trained colon and rectal surgeons, and others experienced in laparoscopic surgery. Numerous publications have attempted to define the number of cases required for adequate learning curve, to safely perform laparoscopic colon and rectal surgery [14-16]. The learning curve required for an experienced surgeon in laparoscopic colectomy to perform laparoscopic rectal resection gained much less attention, and the learning curve required for an institution, or group of surgeons, to master laparoscopic rectal surgery was never measured. Although all attending surgeons in our department had extensive experience in advanced laparoscopic surgery prior to the use of laparoscopy for distal colorectal cancer, it is not impossible that the relatively high complication rate noticed in our study can be partially attributed to the "institutional learning curve", a topic which may gain importance when laparoscopic surgery for rectal cancer becomes standard.

This study represents a series of laparoscopic surgery for distal colorectal cancer performed by various surgeons in an active department of surgery. The main limitation of this study is the lack of a control group. Comparison to historical series would have very limited value, since operative and perioperative techniques as well as oncologic therapy, including the use of preoperative neoadjuvant chemoradiation and advanced adjuvant medications, have largely changed in the past decade. Comparison to patients who underwent open resection in the same time period is also of limited value, since our policy was to offer laparoscopic surgery whenever possible, and significant selection bias is expected comparing this group to those who did not fit for laparoscopic surgery. Thus, prospective controlled trials randomizing patients with rectal cancer to open or laparoscopic surgery are required, to define the role of laparoscopy in these cases. Until the results of such ongoing studies are available, case series can serve as indirect evidences supporting the feasibility and safety of this approach.

Additional limitation of this study is the $75 \%$ long-term follow-up rate. As a territory medical center, part of the patients traveling from other regions of the country for surgery, and are followed locally afterwards. We have made any attempt to localize patients for contact, but a portion of the patients have probably changed contact information or left the country. We can only assume that the $75 \%$ of patients with long-term follow-up do reflect the entire group.

In conclusion, the results of this single institution series suggest that laparoscopic surgery for distal colorectal cancer is feasible, with reasonable conversion rate and adequate oncologic outcome. The complexity of the procedure as well as the level of anastomosis results in relatively high complication rate, which may potentially improve by overcoming the "institutional learning curve", proper patient selection, and further advancement and laparoscopic instruments and techniques. The currently running randomized controlled trials comparing open and laparoscopic surgery for rectal cancer should verify these results, before the laparoscopic approach can be recommended as the standard of care.

\section{CONFLICT OF INTEREST}

None declared.

\section{ACKNOWLEDGEMENT}

None declared.

\section{REFERENCES}

[1] Jayne DG, Guillou PJ, Thorpe H, et al. Randomized trial of laparoscopic- assisted resection of colorectal carcinoma: 3-year results of the UK MRC CLASICC trial group. J Clin Oncol 2007; 25(21): 3061-8.

[2] Milsom JW, de Oliveira OJ, Trenchava KI, et al. Long-term outcomes of patients undergoing curative laparoscopic surgery for mid and low rectal cancer. Dis Colon Rectum 2009; 52(7): 121522.

[3] Staudacher C, Di Paolo S, Tamburini A, Vignali A, Orgenigo E. Total mesorectal excision with laparoscopic approach: 226 consecutive cases. Surg Oncol 2007; 16(Suppl 1): S113-6. 
[4] Feliciotti F, Guerrieri M, Paganini AM, et al. Long-term results of laparoscopic versus open resections for rectal cancer for 124 unselected patients. Surg Endoscopy 2003; 17(10): 1530-5.

[5] Braga M, Frasson M, Zuliani W, Vignalli A, Picorelli N, Di Carlo V. Randomized clinical trial of laparoscopic versus open left colonic resection. Br J Surg 2010; 97(8): 1180-6.

[6] Laurent C, Leblane F, Wutrich P, Scheffler M, Rullier E. Laparoscopic versus open surgery for rectal cancer: long-term oncological results. Ann Surg 2009; 250(1): 54-61.

[7] Fernandez-Cebrian JM, Gil P, Henandez-Granadoz P, et al. Initial surgical experience in laparoscopic total mesorectal excision for middle and lower third rectal cancer: short-term results. Clin Transl Oncol 2009; 11(7): 460-4.

[8] Bretagnol T, Alves A, Rical A, Valleur P, Panis Y. Regtal cancer surgery without mechanical bowel preparation. Br J Surg 2007; 94(10): 1266-71.

[9] Van't Sant HP, Weidema WF. Hop WC, Oostvagel HJ, Contant $\mathrm{CM}$. The influence of mechanical bowel preparation in elective lower colorectal surgery. Ann Surg 2010; 25(1): 59-63.

[10] Zmora O, Lebedyev A, Hoffman A, et al. Laparoscopic colectomy without mechanical bowel preparation. Int. J Colrectal Dis 2006; 21(7): 683-7.
[11] Ulrich AB, Seiber C, Rahbari N, Weitz J, Buchier MW. Diverting stoma after low anterior resection: more arguments in favor. Dis Colon Rectum 2009; 52(3): 412-8.

[12] Eberl T, Jagoditsch M, Kingler A, Tschmelitsch J. Risk factors for anastomotic leakage after resection for rectal cancer. Am J Surg 2009; 196(4): 592-8.

[13] Villanueva-Saenz E, Sierra-Montenegro E, Rojas-Illanes M, PenaRuiz Esparza JP, Martinez Hernandez- Magro P, Bolanos-Badillo LE. Double stapler technique in colorectal surgery. Cir Cir 2008; 76(1): 49-53.

[14] Park IG, Choi GS, Lim KH, Kang BM, Jun SH. Multidimensial analysis of the learning curve for colorectal surgery: lessons from 1000 cases of laparoscopic colorectal surgery. Surg Endosc 2009; 23(4): 839-46.

[15] Li JC, Hon SS, Ng SS, Lee JF, Yui RY, Leung RL. The learning curve for laparoscopic colectomy: experience of a surgical fellow in a university colorectal Unit. Surg Endosc 2009; 23(7): 603-8.

[16] Siani LM, Ferranti F, Di Carlo A, Marzano M, Quintiliani A Laparoscopic total mesorectal excision for extraperitoneal rectal cancer. Oncological outcome at 5 years. Chir Ital 2009; 61(5-6): 585-9.

(C) Lebedyev et al.; Licensee Bentham Open.

This is an open access article licensed under the terms of the Creative Commons Attribution Non-Commercial License (http://creativecommons.org/licenses/ by-nc/3.0/) which permits unrestricted, non-commercial use, distribution and reproduction in any medium, provided the work is properly cited. 